\title{
Role of white-matter pathways in coordinating alpha oscillations in resting visual cortex
}

Hindriks, ${ }^{1, *}$ R., Woolrich, ${ }^{2}$ M., Luckhoo, ${ }^{2,5}$ H., Joensson, ${ }^{3,4}$ M., Mohseni, ${ }^{2}$ H., Kringelbach, ${ }^{3,4}$ M.L., Deco, ${ }^{1,6}$ G.

1. Center for Brain and Cognition, Computational Neuroscience Group, Department of Information and Communication Technologies, Universitat Pompeu Fabra, Roc Boronat 138, Barcelona, 08018, Spain

2. Oxford Centre for Human Brain Activity, University of Oxford, Warneford Hospital, Oxford OX37JX, United Kingdom

3. Department of Psychiatry, University of Oxford, Oxford, Warneford Hospital, Oxford OX37JX, United Kingdom

4. Center of Functionally Integrative Neuroscience (CFIN), Aarhus University, Denmark

5. Centre for Doctoral Training in Healthcare Innovation, Institute of Biomedical Engineering, Department of Engineering Science, University of Oxford, UK

6. Instituci Catalana de la Recerca i Estudis Avanats (ICREA), Universitat Pompeu Fabra, Passeig Llus Companys 23, Barcelona, 08010, Spain

* Corresponding author: Rikkert.Hindriks@upf.edu 


\begin{abstract}
In the absence of cognitive tasks and external stimuli, strong rhythmic fluctuations with a frequency $\sim 10 \mathrm{~Hz}$ emerge from posterior regions of human neocortex. These posterior $\alpha$-oscillations can be recorded throughout the visual cortex and are particularly strong in the calcarine sulcus, where the primary visual cortex is located. The mechanisms and anatomical pathways through which local alpha oscillations are coordinated however, are not fully understood. In this study, we used a combination of magnetoencephalography (MEG), diffusion tensor imaging (DTI), and computational modeling to assess the role of whitematter pathways in coordinating cortical $\alpha$-oscillations. Our findings suggest that primary visual cortex plays a special role in coordinating $\alpha$-oscillations in higher-order visual regions. Specifically, the amplitudes of $\alpha$-sources throughout visual cortex could be explained by propagation of $\alpha$-oscillations from primary visual cortex through white-matter pathways. In particular, $\alpha$-amplitudes within visual cortex correlated with both the anatomical and functional connection strengths to primary visual cortex. These findings reinforce the notion of posterior $\alpha$-oscillations as intrinsic oscillations of the visual system. We speculate they might reflect a default-mode of the visual system during which higher-order visual regions are rhythmically primed for expected visual stimuli by $\alpha$-oscillations in primary visual cortex.
\end{abstract}




\section{Introduction}

The most salient feature of electrical activity in human neocortex in the absence of explicit cognitive tasks are strong $\sim 10 \mathrm{~Hz}$ oscillations [5, 23]. These posterior $\alpha$-oscillations are typically recorded over occipital and posterior-parietal regions and are particularly strong within and around the calcarine fissure - where the primary visual cortex (V1) is located - as well as in the occipito-parietal fissure $[23,11]$. Although initially regarded as functionally irrelevant, evidence is now accumulating that posterior $\alpha$-oscillations do not merely reflect passive idling of visual areas but closely follow allocation of visuo-spatial attention [57, 30, 10]. For example, during anticipatory cue-stimulus intervals, $\alpha$ is suppressed in those regions of primary visual cortex that correspond to attended locations in the visual field and is actively enhanced in unattended or distractor regions [33, 44]. Moreover, these modulations do not only pertain to spatial attention tasks but extend to feature-based attentional processes in higher-order visual areas including the dorsal and ventral projection systems [31,52]. Thus, power fluctuations in posterior $\alpha$-oscillations seem to reflect modulations in cortical excitability, constituting a fundamental mechanism for flexible routing of visual attention $[29,47,53]$. Research on the neuronal mechanisms underlying attention-driven $\alpha$-modulation is expected to benefit from a characterization of the resting-state organization of posterior $\alpha$-oscillations.

Magnetoencephalographic (MEG) recordings in human subjects and local field potential (LFP) recordings in dogs and macaques have shown that posterior $\alpha$ oscillations can be recorded throughout the visual system [38, 49, 23, 11, 6, 7, 53]. In addition to cortical sources of $\alpha$, recordings in behaving dogs and slice preparations of cat lateral geniculate nucleus (LGN) have observed $\alpha$-sources in thalamic nuclei, particularly the LGN and pulvinar [35, 26]. Moreover, the timecourses of sources in LGN and in particular the pulvinar were correlated with various $\alpha$-sources in occipital cortex [36]. Furthermore, EEG-fMRI recordings in humans have found resting-state fluctuations in posterior $\alpha$-power to be correlated with fluctuations in blood-level-oxygenation-level (BOLD) signal throughout the visual system and in several subcortical nuclei $[21,40,19]$. Thus, although posterior $\alpha$-oscillations seem to involve large-scale thalamo-cortical networks, the nature of their involvement remains controversial [51, 32].

In particular, it is unclear if cortical $\alpha$-oscillations reflect independent generators, or if they arise from distant $\alpha$-generators that propagate through whitematter pathways. For example, $\alpha$-oscillations in V1 might be generated within the cortical tissue itself [34], reflect propagated oscillations from LGN [37, 26], which is densely connected to V1 via the optic radiation, or reflect reverberation within thalamo-cortical loop [46, 43]. Similarly, $\alpha$-oscillations in different regions of the visual system might be generated locally or reflect propagated oscillations from distant cortical or thalamic regions. In this study, we assessed the contribution of white-matter pathways in the propagation and coordination of posterior $\alpha$-oscillations. To this end, we combined MEG source-modeling [56], diffusion tensor imaging (DTI) based probabilistic fiber tracking [4], and computational modeling.

We found that the assumption of a single $\alpha$-source in the calcarine sulcus 
(V1) could explain the source-strengths of $\alpha$-oscillations throughout the occipital lobe, medial posterior-parietal cortex and temporal lobes. Furthermore, the source-strengths of $\alpha$-oscillations in these regions correlated with both the functional and anatomical connections to V1, consistent with the assumption of a generator in V1. Although this study does not rule out the possibility that $\alpha$-oscillations are generated throughout the cortex [46, 43, 42], it establishes a central role of V1-connectivity in coordinating $\alpha$-oscillations in the visual system at rest.

\section{Materials and Methods}

\subsection{MEG recordings}

Ten subjects (3 males, 20-39 years old, mean 27.9) underwent an eyes-closed resting-state MEG scan lasting 5 min on an Elekta Neuromag (Elekta Neuromag Oy, Helsinki, Finland). Data preprocessing included signal space separation, denoising with independent component analysis (ICA), source reconstruction and bandpass filtering of the MEG signal. Signal space separation compensated for external interference and sensor artifacts by projection of the MEG data onto a basis set of spherical harmonics. Harmonics corresponding to sources originating from within the sensor array were preserved whilst interfering sources from outside the environment surrounding the sensor array were rejected. The sensor-space MEG data were de-noised using temporal ICA to remove cardiac, $50 \mathrm{~Hz}$ mains and, in some subjects, eye movement artifacts.

Specifically, the data were decomposed into 64 components using fastICA [27] (64 is the rank of the MEG data after signal space separation). Prior to the ICA decomposition, each sensor type was normalized by its smallest eigenvalue to give an unbiased noise estimate across sensor types. Eye-blink, cardiac and mains interference ICA components were manually identified by the combined inspection of spatial topography and time course, kurtosis of the time course, and frequency spectrum for all components. As shown by Mantini et al. [39], it is best to estimate the de-noised sensor data as the subtraction of the artifact components from the raw data. We converted this subtraction into a multiplication via a residual forming matrix. This enabled simultaneous de-noising of the data and correction of the lead fields (via the montage function in SPM8). Each dataset was then co-registered into the Montreal Neurological Institute (MNI) space by registering the canonical MNI template to the Polhemus head shape data. A local spheres forward model [25] was then estimated using the head shape and sensor geometry. Both co-registration and forward model estimation were performed with the matlab SPM8 package (FIL,UCL).

Subsequently, the MEG data were bandpass filtered between 1 and $80 \mathrm{~Hz}$. A LCMV beamformer was used to transform the original sensor time-series into source-space time-series, that is, to reconstruct the activity at the 90 centre locations defined by the AAL brain parcellation. The beamformer uses the forward model and sensor-space covariance matrix to calculate a set of weights which spatially filter the signal so that activity from outside the source is suppressed and the activity from the chosen sources is extracted [56]. The magnetometers 
A

MEG source-strengths $(7-13 \mathrm{~Hz})$

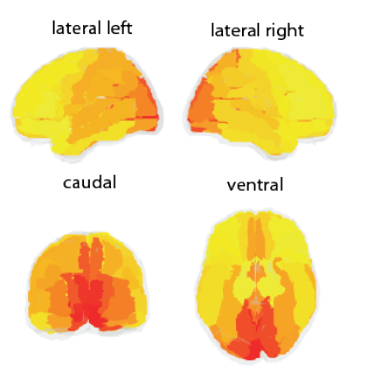

B

DTI probabilistic tractography

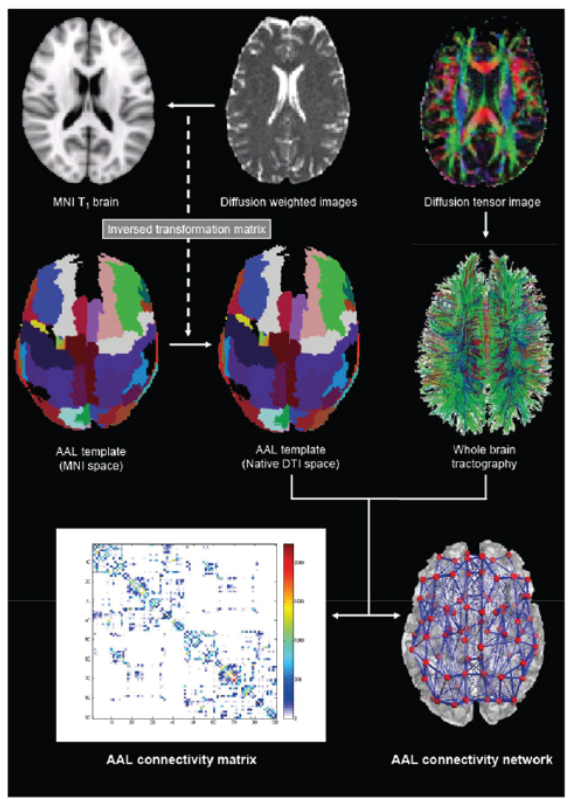

Figure 1: Reconstruction of MEG source-strengths and DTI probabilistic tractography. A. Color-coded standard-deviations of the source-projected MEG time-series in the $\alpha$ frequency band $(7-13 \mathrm{~Hz})$ for all cortical AAL regions. The colorscale ranges from yellow to red. B. Based on MRI images, cerebral cortex is identified and parcellated using the AAL scheme. White-matter tracts are reconstructed from the diffusion tensors using a probabilistic tractography algorithm. The reconstructed tracts between the AAL regions constitute the white-matter network used in this study.

and gradiometers were combined during beamforming by normalizing the data and lead fields for each sensor type by its respective minimum eigenvalue; this effectively gives both classes of sensor equal noise levels. We discarded the reconstructed time-series of all subcortical regions because their lower reliability and subsequently filtered the remaining time-series in the $\alpha$ frequency band $(7-13)$ $\mathrm{Hz}$ and computed their standard-deviations. This resulted in source-strength estimates for all cortical AAL regions, which are shown in Figure 1A.

\subsection{Diffusion tensor imaging}

Diffusion tensor imaging (DTI) data were acquired for 21 healthy participants (10 females, age range 22-45 years) on a Philips Achieva 1.5 Tesla Magnet in Oxford. Diffusion weighted imaging was performed using a single-shot echo planar sequence with coverage of the whole brain. DTI data were acquired with 33 optimal nonlinear diffusion gradient directions $(b=1200 \mathrm{~s} / \mathrm{mm} 2)$ and 1 non-diffusion weighted volume $(b=0)$. The scanning parameters were echo time $(\mathrm{TE})=65 \mathrm{~ms}$ and repetition time $(\mathrm{TR})=9390 \mathrm{~ms}$. For 9 of the 21 participants 
the reconstructed matrix size was $176 \times 176$ with voxel size of $1.8 \times 1.8 \times 2.0 \mathrm{~mm}$, while the remaining 12 participants used a reconstructed matrix of $128 \times 128$ with voxel size of $2.5 \times 2.5 \times 2.5 \mathrm{~mm}$. The construction of structural brain networks consisted of a two-step process. First, the nodes of the network were defined using brain parcellation techniques. Secondly, the connections between nodes (i.e. edges) were estimated using probabilistic tractography (see Figure 1). In the following we outline the details involved in each step.

First, we used the automated anatomical labeling (AAL) template to parcellate the entire brain into 90 cortical and subcortical regions (45 for each hemisphere), where each region represents a node of the brain network [55]. In addition, we created a new AALThal parcellation by combining the AAL atlas with the Oxford Thalamic Connectivity Probability Atlas [4, 2]. The AALThal atlas replaces the thalamus with 7 thalamic subregions and thus has 102 cortical and subcortical regions. The parcellations were conducted in the diffusion MRI native space.The parcellation was conducted in the diffusion MRI native space. We used the Flirt tool (FMRIB, Oxford) [28] to linearly coregister the b0 image in diffusion MRI space to the T1-weighted structural image. The transformed T1-weighted image was then mapped to the T1 template of ICBM152 in MNI space [12]. The resulting transformation was inversed and further applied to warp the Automated Anatomical Labeling (AAL) [55] from MNI space to the diffusion MRI native space, where interpolation used the nearest-neighbor method ensuring the preservation of discrete labeling values.

Secondly, we used the Fdt toolbox in FSL (http://www.fmrib.ox.ac.uk/fsl/, FMRIB, Oxford) to carry out the various processing stages of the diffusion MRI data. The initial preprocessing involved coregistering the diffusion-weighted images to a reference volume using an affine transformation for the correction of head motion as well as eddy current induced image distortion. Following this preprocessing, we estimated the local probability distribution of fiber direction at each voxel [4]. We then used the probtrackx algorithm allowing for automatic estimation of two fiber directions within each voxel, which can significantly improve the tracking sensitivity of non-dominant fiber populations in the human brain [3]. We estimated the connectivity probability by applying probabilistic tractography at the voxel level using a sampling of 5000 streamline fibers per voxel. The connectivity probability from a seed voxel $i$ to another voxel $j$ was defined by the proportion of fibers passing through voxel $i$ that reach voxel $j$ [3]. This was then extended from the voxel level to the region level, i.e. in a brain region consisting of $\mathrm{n}$ voxels, $5000 n$ fibers were sampled. The connectivity probability density $P_{i j}$ from region $i$ to region $j$ is calculated as the number of sampled fibers in region $i$ that connect the two regions divided by $5000 n$, where $n$ is the number of voxels in region $i$. The use of connectivity probability per volume unit, calculated by normalizing the connectivity probability by the number of voxels in each region, is required since the MEG data was projected onto the centres of the AAL regions, rather than onto their entire volumes. For each brain region, the connectivity probability density to each of the other 101 regions was calculated, yielding a $102 \times 102$ matrix $P$. Following [9], we symmetrized the structural matrix by averaging the probability densities $B_{i j}$ and $B_{j i}$ for each pairs of regions $(i, j)$. 
To simplify the structural matrix, we excluded all thalamic segments except for the occipital segment, which projects mainly to the occipital lobe [2]. This segment contains the lateral geniculate nucleus (LGN) which is possibly involved in the generation of posterior $\alpha[35,26]$ as well as the pulvinar, and most likely several intra-laminar nuclei [2]. This yielded a $90 \times 90$ structural probability matrix $B$. We subsequently averaged $B$ over both hemispheres yielding yielding a $45 \times 45$ connectivity matrix. The regions are listed in Table 1 . Justification for this comes from the fact that left and right intra-hemispheric connections are correlated with $\rho=0.92(p=0.0000$, two-sided $t$-test $)$. Furthermore, given the fact that there were no significant differences between MEG $\alpha$-amplitude between homologue cortical areas (the minimum $p$-value over homologue cortical areas obtained from $10^{4}$ hemispherically-randomized amplitudes equals $p=0.7461$ ) we averaged the amplitudes over hemispheres. The hemispheric symmetry of $\alpha$ amplitudes is also evident from a correlation coefficient of $\rho=0.97$ ( $p=0.0000$, two-sides $t$-test) between the amplitudes in left and right hemispheres. Figure 1A shows the topographies of left and right cortical $\alpha$-amplitudes. 


\begin{tabular}{ll}
\hline \hline Region & Abbr \\
\hline Precentral & PreCG \\
Frontal Sup & SFGdor \\
Front Sup Orb & ORBsup \\
Front Mid & MFG \\
Front Mid Orb & ORBmid \\
Front Inf Ope & IFGoperc \\
Front Inf Tri & IFGtriang \\
Front Inf Orb & ORBinf \\
Rolandic Oper & ROL \\
Supp Motor Ar & SMA \\
Olfactory & OLF \\
Front Sup Med & SFGmed \\
Front Med Orb & ORBmid \\
Rectus & REC \\
Insula & INS \\
Cingulum Ant & ACG \\
Cingulum Mid & DCG \\
Cingulum Post & PCG \\
Hippocampus & HPG \\
ParaHippocamp & PHG \\
Amygdala & AMYG \\
Calcarine & CAL \\
Cuneus & CUN \\
Lingual & LING \\
Occipital Sup & SOG \\
Occipital Mid & MOG \\
Occipital Inf & IOG \\
Fusiform & FFG \\
Postcentral & PoCG \\
Parietal Sup & SPG \\
Parietal Inf & IPL \\
SupraMarginal & SMG \\
Angular & ANG \\
Precuneus & PCUN \\
Paracentr Lob & PCL \\
Caudate & CAU \\
Putamen & PUT \\
Pallidum & PAL \\
Thalamus & THA \\
Heschl & HES \\
Temporal Sup & STG \\
Tempr Pol Sup & TPOsup \\
Temporal Mid & MTG \\
Tempr Pol Mid & TPOmid \\
Temporal Inf & ITG \\
& \\
TH
\end{tabular}

Table 1: Descriptions and abbreviations of the AAL regions used in this study. 


\subsection{Dynamical meanfield model}

The electrical activity in individual AAL regions is modeled by adopting a meanfield approach to neuronal dynamics [42, 18]. Specifically, and adopting the same formalism as used in [14, 41], each region is assumed to comprise excitatory and inhibitory neural populations. For cortical and thalamic regions, the excitatory and inhibitory populations correspond to pyramidal (PY) and inhibitory (IN) populations and thalamo-cortical (TC) and reticular (RE) populations, respectively. Membrane excitability is modeled by the function $S(v)=1 /\left(1+e^{-\rho_{1}\left(v-\rho_{2}\right)}\right)$, which converts average membrane potentials into average firing-rates. The parameters $\rho_{2}$ and $\rho_{1}$ denote the average spike-threshold and dispersion of spike-thresholds over the population, respectively. Furthermore, the populations are assumed to integrate incoming spike-rates linearly through synaptic responses parameterized by $h(t)=\epsilon \kappa t e^{-t \kappa}$, where $\epsilon$ and $\kappa$ denote the synaptic efficacy and synaptic rate-constant, respectively. Efficacies and rate-constants depend on the type of synapse (excitatory or inhibitory) and on the types of pre- and post-synaptic populations (PY, IN, TC, or RE). The excitatory and inhibitory populations of a given region are coupled through a number of excitatory $\rightarrow$ inhibitory and inhibitory $\rightarrow$ excitatory synapses, which are denoted by $\gamma_{1}$ and $\gamma_{2}$, respectively.

A

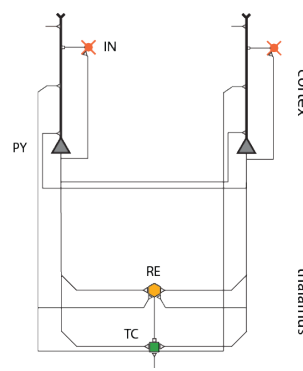

B

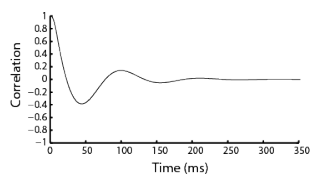<smiles>[CH]1C=C1</smiles>

Figure 2: Computational model of $\alpha$-oscillations. (A) Illustration of the synaptic organization. Local cortical circuits comprise a population of pyramidal (PY) and inhibitory interneurons (IN). Thalamus is modeled by interconnected populations of thalamo-cortical relay (RE) and thalamic reticular (RE) neurons. Local circuitry in cortex and thalamus consists of an inhibitory feedback loop. Cortical regions are coupled via excitatory $\mathrm{PY} \rightarrow \mathrm{PY}$ projections and cortex and thalamus are coupled via excitatory $\mathrm{TC} \rightarrow \mathrm{PY}$ and $\mathrm{PY} \rightarrow \mathrm{TC}$ projections which leave corraterals to reticular neurons (RE). Both PY and TC populations are driven by a constant afferent firing-rate. (B) Autocorrelation function of the excitatory populations for the chosen parameter values (see Appendix A). The autocorrelation function is the inverse Fourier transform of the power spectrum, hence determines the resonance-strength of the population for synaptic inputs of all frequencies. Note that it behaves as a damped oscillation with period $\sim 100 \mathrm{~ms}$, corresponding to $\alpha$-frequency of $\sim 10 \mathrm{~Hz}$. 
The synaptic organization of the model is illustrated in Figure 2A. Corticocortical pathways are modeled by excitatory PY $\rightarrow$ PY projections and the thalamo-cortico-thalamic loop consists of excitatory thalamo-cortical projections TC $\rightarrow$ PY and excitatory cortico-thalamic projections PY $\rightarrow$ TC which leave collaterals PY $\rightarrow$ RE to the reticular population. The coupling strengths are assumed to be proportional to the corresponding entries of the connectivity probability density matrix $B$. In addition, the excitatory populations in both cortex and thalamus are driven by a constant afferent firing-rate which models non-specific background activity. We use independent global coupling strengths $\mathrm{K}_{1}$ and $\mathrm{K}_{2}$ for cortico-cortical and cortico-thalamo-cortical connections, respectively. Thus, $\mathrm{K}_{1}$ corresponds to the overall strength of cortico-cortical connections and $\mathrm{K}_{2}$ to the overall strength of feedback within the cortico-thalamocortical loop. They are incorporated into the model by using them as weights in the connectivity density matrix $B$. Following previous modeling studies $[20,15,9,16] \mathrm{K}_{1}$ and $\mathrm{K}_{2}$ are considered free parameters and are used to tune the model. Source-projected MEG signals are assumed to be proportional to the average dendritic activity of the pyramidal populations in the corresponding cortical AAL regions $[42,18]$. The model equations and parameter values are given in Table 2 in Appendix A.

The parameter values determining the dynamics of cortical and thalamic regions were chosen to be identical and such that the excitatory populations had a stable equilibrium voltage and resonated at a frequency $\sim 10 \mathrm{~Hz}$. Hence we assume that the local circuitry of all regions is tuned to resonate at $\alpha$-frequency when driven by excitatory synaptic input. Figure 2B provides an illustration. We investigated two scenario's for $\alpha$-generation, namely, local generation in V1 and distributed generation troughout the cortex. An $\alpha$-generator in primary visual cortex was modeled by driving the excitatory population by excitatory synaptic input, modeled as white-noise with standard deviation $\sigma=1 \mathrm{~s}^{-1}$. Distributed $\alpha$-generation were modeled by setting $\sigma=1 \mathrm{~s}^{-1}$ for all cortical regions.

\section{Results}

\subsection{Dynamical workingpoint}

To obtain a dynamical workingpoint for the model, we first determined the models' stability boundary in the plane spanned by the cortico-cortical and thalamo-cortical connection-strengths $\mathrm{K}_{1}$ and $\mathrm{K}_{2}$, respectively. In terms of dynamics, restricting the workingpoint to the stable region means that we assume resting-state $\alpha$-oscillations to emerge from stochastic perturbations of a stable equilibrium state, in agreement with empirical studies [54,24] and in line with modeling studies on fMRI resting-state dynamics [20,17]. To determine the stability boundary, we compute the models' Jacobian at the steady-state and numerically calculate its eigenvalues (see Appendix B). Each eigenvalue $\lambda$ is complex-valued and describes the resonance behavior of one of the statevariables. Specifically, the response of the state-variable upon an instantaneous perturbation is an exponentially damped oscillation $e^{\lambda}$, where the damping-rate $\operatorname{Re}(\lambda)$ and frequency $\operatorname{Im}(\lambda)$ describe the characteristic time-scale and angu- 
lar frequency of the response. The stable regime corresponds to the values of $\left(\mathrm{K}_{1}, \mathrm{~K}_{2}\right)$ for which the damping-rate $\operatorname{Re}(\lambda)<0$ for all state-variables. Figure $3 \mathrm{~A}$ and $\mathrm{B}$ show color-coded damping-rate and frequency of the least-damped eigenvalue as a function of $\left(\mathrm{K}_{1}, \mathrm{~K}_{2}\right)$. The boundary in the images corresponds to the stability boundary. Note that approaching the stability boundary leads to increased alpha frequency. When crossed, the system destabilizes through a supercritical Hopf bifurcation, giving rise to self-sustained $\alpha$-oscillations, a scenario that is more likely to be related to epileptic dynamics, in particular tonic-clonic seizures $[45,8]$.

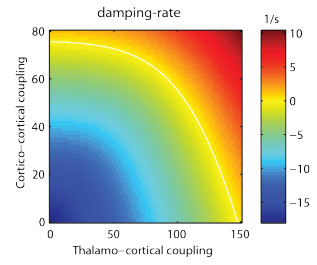

C

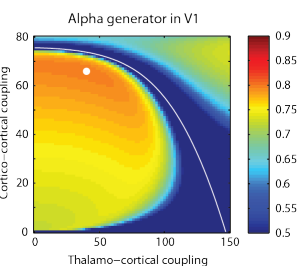

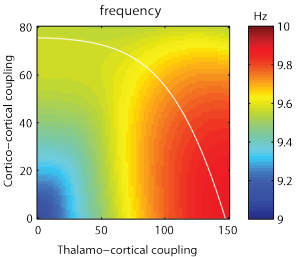

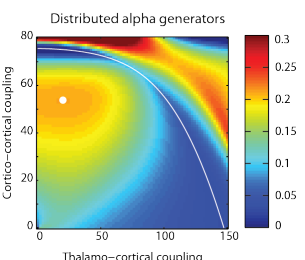

Figure 3: Thalamo-cortical stability and dynamical workingpoint. A Dampingrate of the least-damped eigenvalue as a function of cortico-cortical and thalamocortical coupling strength $\mathrm{K}_{1}$ and $\mathrm{K}_{2}$. B Frequency of the least-damped eigenvalue as a function of cortico-cortical and thalamo-cortical coupling strength $\mathrm{K}_{1}$ and $\mathrm{K}_{2}$. C. Pearson correlation coefficients between the observed $\alpha$-amplitudes and those predicted by the model by assuming a single $\alpha$-generator in the Calcarine sulcus $(\mathrm{V} 1)$. The correlation is maximal at $\left(\mathrm{K}_{1}^{*}, \mathrm{~K}_{2}^{*}\right)=(66,40)$ and is denoted by a white dot. D. Pearson correlation coefficients between the observed $\alpha$-amplitudes and those predicted by the model by assuming $\alpha$ generators to be distributed throughout the cortex. The correlation is maximal at $\left(\mathrm{K}_{1}^{*}, \mathrm{~K}_{2}^{*}\right)=(66,40)$ and is denoted by a white dot. The correlation coefficients in the right-hand-side figure were predicted by the model in which $\alpha$-generators were assumed to be distributed throughout the cortex. The correlation is maximal around $\left(\mathrm{K}_{1}^{*}, \mathrm{~K}_{2}^{*}\right)=(53,20)$ and is denoted by a white dot. Note the difference in colorscaling between $\mathrm{C}$ and $\mathrm{D}$. In all figures, $\mathrm{K}_{1}$ and $\mathrm{K}_{2}$ range from 0 to 80 and from 0 to 150 , respectively, in steps of 1 and 2, respectively and the curved white boundary coincides with the (linear) stability boundary. 
To find a dynamical workingpoint $\left(\mathrm{K}_{1}^{*}, \mathrm{~K}_{2}^{*}\right)$, we placed a generator in primary visual cortex and computed the Pearson correlation coefficient between the predicted and observed cortical $\alpha$-amplitudes, that is, between the standarddeviations of the modeled time-series and the corresponding source-projected MEG time-series filtered in the $\alpha$ frequency band. We did this as a function of $\left(\mathrm{K}_{1}, \mathrm{~K}_{2}\right)$ in the entire stable regime. The predicted amplitudes were computed semi-analytically (see Appendix $\mathrm{C}$ ). The correlation coefficients reached a local maximum of $\rho=0.80$ at $\left(\mathrm{K}_{1}^{*}, \mathrm{~K}_{2}^{*}\right)=(66,40)$, which we chose as the dynamical working point. The dynamical workingpoint is designated in Figure $3 \mathrm{C}$ by a white dot. Importantly, the fact that the model performs best well away from the horizontal and vertical boundaries, which correspond to the absence of cortico-cortical and thalamo-cortical connections respectively, suggests the involvement of both cortico-cortical as well as thalamo-cortical pathways in shaping the distribution of cortical $\alpha$-amplitudes. Also note that the model performs best for a value of $\left(\mathrm{K}_{1}, \mathrm{~K}_{2}\right)$ that lies in the interior of the stability region, as opposed to a best fit on the instability boundary. This shows that the chosen workingpoint corresponds to a well-defined best fit.

Since in the above simulations, primary visual cortex displayed much larger oscillations than other regions - in contrast to the observed oscillations - we excluded primary visual cortex before computing the correlation coefficients. This discrepancy between model and data might be caused be passive propagation of the magnetic field generated by cortical sources leading to an offset in the amplitudes of the source-projected MEG signals which is absent in the simulated data. Alternatively, it could be that a single generator in V1 is too restricted and that the data may be better explained by assuming multiple $\alpha$-generators. To test this possibility, we placed a generator in every cortical region and repeated the above assesment of how well the observed $\alpha$-amplitudes are predicted by the model. The result is shown in Figure 3D. The figure shows that although there is a well-defined maximum in model performance, the maximal correlation coefficient is low $(\rho=0.21)$.

Figure 3C and 3D show that in both model simulations, the best fit is obtained for non-zero cortico- and thalamo-cortical coupling strengths, which suggest involvement of both types of pathways in coordinating cortical $\alpha$-oscillations. However, when the workingpoints (white dots) are projected to the corticocortical axis however, the correlation with the data remains high, while projection to the thalamo-cortical axis yields substantially lower correlations. Thus, in both simulations, cortico-cortical pathways contribute more to structuring $\alpha$-amplitudes than thalamo-cortical connections do. This is in agreement with the findings reported in [36] using local field potentials simultaneously recorded from visual cortex and thalamus. Using partial coherence analysis, it was found that cortico-cortical connections contributed more to the coherence between $\alpha$ oscillations in different cortical regions than thalamo-cortical connections did.

\subsection{Spatial extent of posterior alpha oscillations}

Figure $4 \mathrm{~A}$ shows the scatterplot of the observed $\alpha$-amplitudes versus those predicted by the model in the chosen workingpoint $\left(\mathrm{K}_{1}^{*}, \mathrm{~K}_{2}^{*}\right)$. They are correlated with Pearson correlation coefficient $\rho=0.80$. To determine the spatial ex- 
tent of posterior $\alpha$-oscillations, we selected the regions that were anatomically connected to primary visual cortex with a connection strength $\geq h$ and computed the Pearson correlation coefficient between the predicted and observed amplitudes within the selected regions. The regions were determined by computing the correlation coefficients as a function of $h$ and subsequently selecting the value of $h$ for which the correlation coefficient attained a local maximum. This gave a threshold of $h=0.03$ (see SI Figure 3). Propagation was found to pervade the entire occipital lobe, with extensions to posterior-parietal and temporal regions. Specifically, $\alpha$ propagated to the inferior, medial, and superior occipital gyri (IOG, MOI, SOG), cuneus (CUN), lingual gyrus (LING), posterior cingulate gyrus (PCG), hippocampal gyrus (HPG), parahippocampal gyrus (PHG), fusiform gyrus (FFG), and precuneus (PCUN) and are displayed on a glass brain in Figure 4B.

A

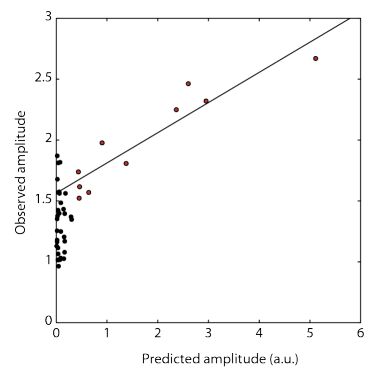

B

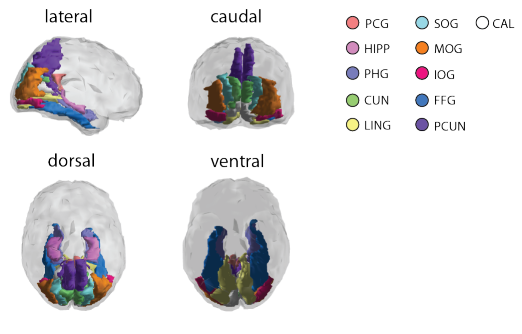

Figure 4: Model performance and spatial extent of posterior $\alpha$-oscillations. A. Scatterplot of observed versus predicted $\alpha$-amplitudes for the model in the workingpoint $\left(\mathrm{K}_{1}^{*}, \mathrm{~K}_{2}^{*}\right)$. The variance of the predicted amplitudes is scaled to unity. The regions to which $\alpha$-oscillations generated in the primary visual cortex propagate are colored red. B. Glass-brain views of the posterior $\alpha$-network. It comprises the inferior, medial, and superior occipital gyri (IOG, MOI, SOG), cuneus (CUN), lingual gyrus (LING), posterior cingulate gyrus (PCG), hippocampal gyrus (HPG), parahippocampal gyrus (PHG), fusiform gyrus (FFG), and precuneus (PCUN). Colors are insignificant and merely serve to delineate the different regions. 
In the sequel, we collectively refer to these regions as the posterior $\alpha$-network. Note that the posterior alpha network roughly comprises visual cortical regions, including the dorsal and ventral projection systems, and thus reinforces the notion of posterior $\alpha$-oscillations as intrinsic oscillations of the visual system. The Pearson correlation coefficient between predicted and observed amplitudes within the posterior $\alpha$-network was $\rho=0.93$, which demonstrates the model's ability to reproduce the relative $\alpha$-amplitudes. The amplitudes within the visual system are denoted by red dots in Figure 4A. Interestingly, the regions comprising the posterior alpha network largely coincide with those in which resting-state BOLD-fluctuations are (negatively) correlated with simultaneously recorded posterior $\alpha$-power fluctuations [19]. This might suggest that restingstate BOLD-fluctuations within the visual system are driven by fluctuations in primary visual cortex.

\subsection{Involvement of primary visual pathways}

If the hypothesis that posterior $\alpha$-oscillations propagate from primary visual cortex to higher-order visual areas through excitatory white-matter pathways is correct, one might suspect a positive correlation between $\alpha$-amplitudes of regions within the posterior $\alpha$-network and corresponding structural connectionstrengths to primary visual cortex. Figure 5A, left-hand-side, shows the scatterplot between predicted posterior $\alpha$-amplitudes and corresponding connectionstrengths, indeed predicting a positive correlation. The correlation coefficient between observed $\alpha$-amplitudes and connection-strengths to primary visual cortex was $\rho_{\alpha, \mathrm{sc}}=0.95(p<0.0001$, two-sided $t$-test $)$, thereby confirming this prediction.

To factor out spurious correlations due to (weak) dependence of observed $\alpha$ amplitudes and connection-strengths to primary visual cortex on Euclidean distance to primary visual cortex $\left(\rho_{\alpha, \text { dist }}=-0.80, p=0.0050\right.$ and $\rho_{\text {sc }, \text { dist }}=$ $-0.71, p=0.0204$, two-sided $t$-tests), which also holds for the predicted amplitudes, we conditioned $\rho_{\alpha, \mathrm{sc}}$ on Euclidean distance, which yielded a partial correlation coefficient of $\rho_{\alpha, \mathrm{sc} \mid \mathrm{dist}}=0.89(p=0.0012$, two-sided $t$-test), which shows that the correlation between $\alpha$-amplitude and connection-strength cannot be explained by their common dependence of distance to primary visual cortex. In contrast, $\rho_{\alpha, \text { dist }}$ vanished when conditioned on connection-strength $\left(\rho_{\alpha, \mathrm{dist} \mid \mathrm{sc}}=-0.57, p=0.1112\right.$, two-sided $t$-test $)$, which shows that the correlation between $\alpha$-amplitude and distance reflects the dependence of structural connection-strength to primary visual cortex on distance. 

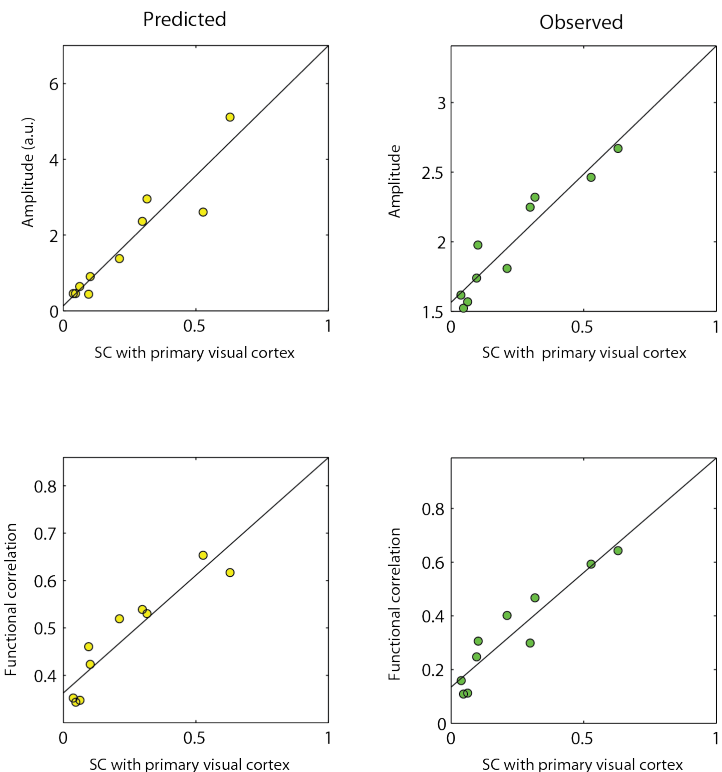

Figure 5: Correlations between $\alpha$-amplitude and structural and functional connections. A. Scatterplot of the predicted (left) and observed (right) $\alpha$ amplitudes of regions within the visual $\alpha$-network versus corresponding structural connection-strengths with primary visual cortex. B. Scatterplots of predicted (left) and observed (right) $\alpha$-amplitudes of regions within the visual $\alpha$ network versus corresponding functional connection-strengths with primary visual cortex. Observed functional correlations were quantified by bandlimited power (BLP) correlations, that is, by pair wise Pearson correlation coefficients between the Hilbert envelopes of the source-projected MEG signals. Superimposed are regression lines obtained from a linear least-squares fit. Predicted amplitudes have been normalized to unit standard-deviation.

If $\alpha$-oscillations propagate from primary visual cortex higher-order visual areas, besides a correlation between $\alpha$-amplitudes and corresponding connectionstrengths to primary visual cortex, we might expect a correlation between $\alpha$ amplitudes and corresponding functional correlations with primary visual cortex. This is illustrated in Figure 5B, left-hand-side, which shows that the model indeed predicts the existence of such a correlation. Functional correlations from the model were measured by the Pearson correlation coefficients between the theoretical time-series and were computed semi-analytically from the linearized model equations (see Appendix D). Figure 5B, right-hand-side, shows that this prediction holds for the data as well $\left(\rho_{\mathrm{fc}, \mathrm{sc}}=0.94, p=0.0001\right.$, two-sided $t$-test). Observed functional correlations were quantified by the bandlimited power (BLP) correlations between the source-projected MEG signals, that is, by the Pearson correlation coefficients between the corresponding Hilbert envelopes. Although, the predicted functional correlations were quantified differently than in the model, allowing for a semi-analytical computation without residing to numerical simulations, the two kinds of correlations are approxi- 
mately proportional within the model, justifying their use in linear correlation analysis. To factor out spurious correlations due to the negative dependence of functional correlation on distance $\left(\rho_{\mathrm{fc}, \text { dist }}=-0.87, p=0.001\right.$, two-sided $t$-test $)$ we conditioned the correlation on Euclidean distance. The partial correlation remained significant $\left(\rho_{\mathrm{fc}, \mathrm{sc} \mid \mathrm{dist}}=0.92, p=0.0004\right.$, two-sided $t$-test $)$ showing that $\rho_{\mathrm{fc}, \mathrm{sc}}$ can only partially be explained by a common dependence of functional correlation and connection-strength of distance. 


\section{Discussion}

In this study we combined MEG source-modeling [56], DTI probabilistic tractography, connectivity-based thalamic segmentation $[4,2,3]$, and computational modeling to investigate the role of white-matter pathways in coordinating $\alpha$ oscillations in human cortex at rest. We focused on two scenario's for the generation of $\alpha$-oscillations, namely, local generation within the Calcarine sulcus (V1) as suggested by MEG inverse-modeling studies [49, 23, 11] and distributed generation of $\alpha$-oscillations throughout the cortex. Both scenario's suggested that $\alpha$-oscillations propagate through cortico-cortical as well as thalamo-cortical pathways, although cortico-cortical pathways seemed to play a larger role, in line with local field potential recordings in dogs [36]. The assumption of a single $\alpha$-generator in V1 however, led to a much better fit with the data than the distributed scenario. While this finding does not rule out the existence of distributed $\alpha$-generators, it at least suggests that V1 plays a central role in coordinating posterior $\alpha$-oscillations. Furthermore, the models' ability of predicting MEG source-strengths of $\alpha$-oscillations was limited to the occipital lobe and parts of the temporal and medial-posterior parietal lobe, namely the inferior, medial, and superior occipital gyri, cuneus, lingual gyrus, posterior cingulate gyrus, hippocampal gyrus, parahippocampal gyrus, fusiform gyrus, and precuneus. This reinforces the notion that posterior $\alpha$-oscillations are intrinsic to the visual system. Both the anatomical and functional connections to primary visual cortex correlated with the source-strengths of $\alpha$-oscillations within this network, as predicted by the model.

Equivalent-dipole modeling of resting-state MEG $\alpha$-oscillations has shown that the strongest $\alpha$-sources are located in the calcarine sulcus (primary visual cortex) and occipito-parietal fissure. The relative source-strengths however, differ from subject to subject $[49,23,11]$. In line with these observations, the groupaveraged MEG source-strength of our data was highest in the calcarine sulcus, followed by the cuneus, which extends into the medial part of the posterior wall of the occipito-parietal fissure. However, both in the data and model, cortical source-strengths only correlated with white-matter connections to the calcarine sulcus and not with the cuneus. Since calcarine was most strongly connected to the cuneus, this suggests that propagation of oscillations within the calcarine sulcus might contribute to the strong $\alpha$-sources within the occipito-parietal fissure.

Since for all subjects, the $\alpha$-amplitudes in the calcarine sulcus and occipitoparietal fissure were of similar magnitude, we suspect that the estimated MEG source-strengths are attenuated by partial cancelation of coherent oscillations in opposite banks of the calcarine sulcus $[49,23,42]$. This remains speculative however, and the use of MEG alone might not be sufficient to provide a definite answer, because MEG is primarily sensitive to the tangential component of current sources [22]. A more complete picture of the organization of cortical $\alpha$-sources likely has to come from combined EEG-MEG source-imaging methods [1] as they exploit the complementary information of MEG and EEG, which is primarily sensitive to the radial component of current sources. In addition to combining EEG and MEG, the inverse-solution space might be reduced further by assuming the current densities to be restricted to the cortical surface and 
their orientation to be perpendicular to it [13]. This would, however, require using a more fine-grained parcellation of the cortical surface and higher-resolution structural matrices.

Several studies have shown that posterior $\alpha$-oscillations can be actively enhanced or suppressed in visual attention paradigms in a retinotopically specific way $[57,33,44,30,10]$ both in primary as well as in higher-order visual areas $[31,52]$. Based on our results, we might speculate that in resting-state, propagation of $\alpha$ from lower to higher-order visual areas might provide a default organization of visual cortex in which modulation of $\alpha$ in primary visual cortex induces coherent modulations of $\alpha$ in higher-order visual areas through retinotopically-organized pathways. Through such a mechanism, retinotopical-specific priming of primary visual cortex might automatically prime corresponding locations in higher-order areas. Such a mechanism could coexest together with top-down modulating signals, which are known or be broadcasted by several regions, most notably frontal and parietal areas [33] as well as several subcortical nuclei [19], in particular the pulvinar [50, 48], whose connections to visual cortex are known to be organized in a retinotopic way [50]. Most likely, the spatio-temporal organization of posterior $\alpha$ emerges from coordinated modulating influences through both top-down and bottom-up cortico-cortical and pulvinar-cortical pathways.

The current study is limited in several respects that need to be addressed in subsequent studies. First, thalamic voxels were classified based on the abundance of their structural connections with the major anatomical regions in cerebral cortex, namely occipital, parietal, temporal, frontal, motor, pre-motor, and somato-sensory regions, hence did not allow delineation of the lateral geniculate nucleus (LGN) and the isolation of higher-order visual thalamic nuclei [2]. As a consequence, our findings do not allow to distinguish the different scenario's of $\alpha$-generation, namely generation in cortical [34] or thalamic $[37,26]$ tissue, or reverberation within thalamo-cortical loop [46, 43], nor allows to assess the role of higher-order thalamic nuclei in modulation of cortical $\alpha$-oscillations $[19,48]$. A more fine-grained structural segmentation of thalamus might allow for a more detailed study of the role of different thalamic nuclei in generation and modulation of cortical $\alpha$. Second, the MEG recordings and MRI scans were obtained from different subject groups. Remarkably, the observed correlations between the anatomical and functional organization of posterior $\alpha$-oscillations on the group-level reflects their robustness. Paired MEG-DTI recordings obtained in a subsequent study will allow further validation of the findings reported in the current study.

Third, the predicted and observed $\alpha$-amplitudes differ in two respects. The first difference is the existence of an off-set in the observed amplitudes which is absent in the predicted amplitudes (see Figure 4A). The second difference is that the predicted amplitude in V1 is much higher than the observe amplitude in V1. Although these differences could be explained by passive propagation of the generated magnetic field, a test of this possibility requires the use of a high-resolution surface-based cortical atlas, rather then the low-resolution volumetric atlas used in this study. An alternative explanation is that there exist multiple $\alpha$-generators, leading to a smoother spatial arrangement of the amplitudes $[46,45]$. Assuming multiple $\alpha$-generators in the current model, however, 
led not lead to a good fit with the data (see Figure 3D). Thus, although a definite answer to the issue of localized versus distributed generation of cortical $\alpha$-oscillations has to come from future research, our study has suggested a central role of V1-connectivity in coordinating $\alpha$-oscillations in the visual system at rest. 


\section{Acknowledgements}

The authors thank Ole Jensen from the Donders Institute for Brain, Cognition, and Behaviour, Nijmegen, The Netherlands and Wessel van Wieringen from the VU University Amsterdam, The Netherlands for valuable conversations regarding this manuscript.

GD was supported by the ERC Advanced Grant: DYSTRUCTURE (n. 295129), by the Spanish Research Project SAF2010-16085 and by the CONSOLIDERINGENIO 2010 Program CSD2007-00012, and the FP7-ICT BrainScales. The authors declare no competing financial interests. 


\section{References}

[1] S. Baillet, L. Garnero, G. Marin, and J.-P. Hugonin. Combined MEG and EEG source imaging by minimization of mutual information. IEEE transactions on biomedical engineering, 46(5), 1999.

[2] T. E. J. Behrens, M. W. Woolrich, S. M. Smith, P. A. Boulby, G. J. Barker, E. L. Sillery, K. Sheehan, O. Ciccarelli, A. J. Thompson, J. M. Brady, and P. M. Matthews. Non-invasive mapping of connections between human thalamus and cortex using diffusion imaging. Nature, 6(7), 2003.

[3] T.E.J. Behrens, H.J. Berg, S. Jbabdi, M.F.S. Rushworth, and M.W. Woolrich. Probabilistic diffusion tractography with multiple fibre orientations : What can we gain ? NeuroImage, 34:144-155, 2007.

[4] T.E.J. Behrens, M.W. Woolrich, M. Jenkinson, R.G. Nunes, S. Clare, P.M. Matthews, J M Brady, and S M Smith. Characterization and Propagation of Uncertainty in Diffusion-Weighted MR Imaging. Magnetic Resonance in Medicine, 50:1077-1088, 2003.

[5] H. Berger. Uber das Elektrenkephalogramm des Menschen. European Archives of Psychiatry and Clinical Neuroscience, 278(1875), 1929.

[6] A. Bollimunta, Y. Chen, C.E. Schroeder, and M. Ding. Neuronal mechanisms of cortical alpha oscillations in awake-behaving macaques. The Journal of neuroscience, 28(40):9976-88, October 2008.

[7] Anil Bollimunta, Jue Mo, Charles E Schroeder, and Mingzhou Ding. Neuronal mechanisms and attentional modulation of corticothalamic $\alpha$ oscillations. The Journal of neuroscience, 31(13):4935-43, March 2011.

[8] M. Breakspear, J.A. Roberts, J.R. Terry, S. Rodrigues, N. Mahant, and P.A. Robinson. A unifying explanation of primary generalized seizures through nonlinear brain modeling and bifurcation analysis. Cerebral cortex (New York, N.Y. : 1991), 16(9):1296-313, September 2006.

[9] Joana Cabral, Etienne Hugues, Olaf Sporns, and Gustavo Deco. Role of local network oscillations in resting-state functional connectivity. NeuroImage, 57:130-139, 2011.

[10] A. Capilla, J.-M. Schoffelen, G. Paterson, G. Thut, and J. Gross. Dissociated $\alpha$-Band Modulations in the Dorsal and Ventral Visual Pathways in Visuospatial Attention and Perception. Cerebral cortex (New York, N.Y. : 1991), pages $1-12,2012$.

[11] C. Ciulla, T. Takeda, and E. Hiroshi. MEG Characterization of Spontaneous Alpha Rhythm in the Human Brain. 11(3):211-222, 1999.

[12] D. Collins, P. Neelin, T. Peters, and A. C. Evans. Automatic 3D intersubject registration of $\mathrm{MR}$ volumetric data in standardized Talairach space. Journal of Computer Assisted Tomography, 18:192-205, 1994.

[13] A.M. Dale and M.I. Sereno. Improved localization of cortical activity by combining EEG and MEG with MRI cortical surface reconstruction: A linear approach. Journal of Cognitive Neuroscience, 5(2):162-176, 1993. 
[14] O. David, S.J. Kiebel, L.M. Harrison, J. Mattout, J.M. Kilner, and K.J. Friston. Dynamic causal modeling of evoked responses in EEG and MEG. NeuroImage, 30(4):1255-72, May 2006.

[15] G. Deco, V. Jirsa, A.R. Mcintosh, O. Sporns, and R. Kotter. Key role of coupling, delay, and noise in resting brain fluctuations. Proceedings of the National Academy of Sciences of the United States of America, 106(25):10302-10307, 2009.

[16] G. Deco and V.K. Jirsa. Ongoing Cortical Activity at Rest : Criticality , Multistability, and Ghost Attractors. The Journal of Neuroscience, 32(10):3366-3375, 2012.

[17] G. Deco, A. Ponce-Alvarez, D. Mantini, G. L. Romani, P. Hagmann, and M. Corbetta. Resting-State Functional Connectivity Emerges from Structurally and Dynamically Shaped Slow Linear Fluctuations. Journal of Neuroscience, 33(27):11239-11252, 2013.

[18] Gustavo Deco, Viktor K Jirsa, Peter A Robinson, Michael Breakspear, and Karl Friston. The Dynamic Brain : From Spiking Neurons to Neural The dynamic brain: from spiking neurons to neural masses and cortical fields. PLoS computational biology, 4(8), 2008.

[19] B. Feige, K. Scheffler, F. Esposito, F. D. Salle, E. Seifritz, K. Scheffler, and F. Di. Cortical and Subcortical Correlates of Electroencephalographic Alpha Rhythm Modulation. Journal of neurophysiology, 93:2864-2872, 2005.

[20] A. Ghosh, Y. Rho, A.R. McIntosh, R. Kotter, and V.K. Jirsa. Noise during Rest Enables the Exploration of the Brain s Dynamic Repertoire. PLoS computational biology, 4(10), 2008.

[21] R.I. Goldman, J.M. Stern, J. Engel, and M.S. Cohen. Simultaneous EEG and fMRI of the alpha rhythm. Neuroreport, 13(18):2487-92, December 2002 .

[22] M. Hamalainen, R. Hari, R. J. Ilmoniemi, J. Knuutila, and O. V. Lounasmaa. Magnetoencephalographytheory, instrumentation, and applications to noninvasive studies of the working human brain. Reviews Modern Physics, $65,1993$.

[23] R. Hari and R. Salmelin. Human cortical oscillations : a neuromagnetic view through the skull. Trends in neurosciences, 20(1):44-49, 1997.

[24] R. Hindriks, F. Bijma, B.W. van Dijk, Y.D. van der Werf, E.J.W. van Someren, and A.W. van der Vaart. Dynamics underlying spontaneous human alpha oscillations: A data-driven approach. NeuroImage, 57(2):440 51, July 2011.

[25] M.X. Huang, J.C. Mosher, and R.M. Leahy. A sensor-weighted overlappingsphere head model and exhaustive head model comparison for MEG. Phys. Med. Biol., 44:423-440, 1999. 
[26] S.W. Hughes, M. Lörincz, D.W. Cope, K.L. Blethyn, K.A. Kékesi, H.R. Parri, G. Juhász, and V. Crunelli. Synchronized oscillations at alpha and theta frequencies in the lateral geniculate nucleus. Neuron, 42(2):253-68, April 2004.

[27] A. Hyvarinen. Fast and Robust Fixed-Point Algorithms for Independent Component Analysis. IEEE Transactions on Neural Networks, 10(3):626$634,1999$.

[28] M. Jenkinson, P. Bannister, M. Brady, and S. Smith. Improved Optimization for the Robust and Accurate Linear Registration and Motion Correction of Brain Images. NeuroImage, 17:825-841, 2002.

[29] O. Jensen, J. Gelfand, J. Kounios, and J. Lisman. Oscillations in the alpha band $(9-12 \mathrm{~Hz})$ increase with memory load during retention in a short-term memory task. Cerebral cortex (New York, N.Y. : 1991), 12(8):877-82, August 2002.

[30] O. Jensen, A. Mazaheri, and O. Box. Shaping functional architecture by oscillatory alpha activity : gating by inhibition. frontiers in Human Neuroscience, 4(186):1-8, 2010.

[31] D. Jokisch and O. Jensen. Modulation of Gamma and Alpha Activity during a Working Memory Task Engaging the Dorsal or Ventral Stream. Journal of Neuroscience, 27(12):3244-3251, 2007.

[32] F.N. Karameh, M.A. Dahleh, E.N. Brown, and S.G. Massaquoi. Modeling the contribution of lamina 5 neuronal and network dynamics to low frequency EEG phenomena. Biological cybernetics, 95(4):289-310, October 2006.

[33] S. P. Kelly, E. C. Lalor, R. B. Reilly, J. J. Foxe, P. Simon, E. C. Lalor, and R. B Reilly. Increases in Alpha Oscillatory Power Reflect an Active Retinotopic Mechanism for Distracter Suppression During Sustained Visuospatial Attention. Journal of neurophysiology, 95:3844-3851, 2006.

[34] D.T.J. Liley, P.J. Cadusch, and J.J. Wright. A continuum theory of electrocortical activity. Neurocomputing, 26-27:795-800, June 1999.

[35] F H Lopes da Silva. Essential differences between alpha rhythms and barbiturate spindles: spectra and thalamo-cortical coherences. Electroencephalography and Clinical Neurophysiology, 35:641-645, 1973.

[36] F. H. Lopes Da Silva, J. E. Vos, J. Mooibroek, and A. van Rotterdam. Relative contributions of intracortial and thalamo-cortical processes in the generation of alpha rhythms, revealed by partial coherence analysis. Electroencephalography, 50:449-456, 1980.

[37] F.H. Lopes da Silva, A. Hoeks, H. Smits, and L.H. Zetterberg. Model of brain rhythmic activity. Kybernetic, 15:27-37, 1974.

[38] F.H. Lopes Da Silva and W. Storm van Leeuwen. The cortical source of the alpha rhythm. Neuroscience letters, 6(2-3):237-241, 1977. 
[39] D. Mantini, S.D. Penna, L. Marzetti, F. Pasquale, de, V. Pizzella, M. Corbetta, and G.L. Romani. A Signal-Processing pipeline for magnetoencephalography resting-state networks. Brain Connectivity, 1(1), 2011.

[40] M. Moosmann, P. Ritter, I. Krastel, A. Brink, S. Thees, F. Blankenburg, B. Taskin, H. Obrig, and A. Villringer. Correlates of alpha rhythm in functional magnetic resonance imaging and near infrared spectroscopy. NeuroImage, 20:145-158, 2003.

[41] R.J. Moran, S.J. Kiebel, K.E. Stephan, R/B. Reilly, J. Daunizeau, and K.J. Friston. A neural mass model of spectral responses in electrophysiology. NeuroImage, 37(3):706-20, September 2007.

[42] P.L. Nunez and R. Srinivasan. Electric fields of the brain. The neurophysics of EEG. 2006.

[43] C.J. Rennie, P.A. Robinson, and J.J. Wright. Unified neurophysical model of EEG spectra and evoked potentials. Biological cybernetics, 86(6):457-71, June 2002.

[44] T. A. Rihs, C. M. Michel, and G. Thut. Mechanisms of selective inhibition in visual spatial attention are indexed by a -band EEG synchronization. European Journal of Neuroscience, 25:603-610, 2007.

[45] P. Robinson, C. Rennie, and D. Rowe. Dynamics of large-scale brain activity in normal arousal states and epileptic seizures. Physical Review E, 65(4):1-9, April 2002.

[46] P. Robinson, C. Rennie, J. Wright, H. Bahramali, E. Gordon, and D. Rowe. Prediction of electroencephalographic spectra from neurophysiology. Physical Review E, 63(2):19-33, January 2001.

[47] V. Romei, V. Brodbeck, and C. Michel. Spontaneous Fluctuations in Posterior a -Band EEG Activity Reflect Variability in Excitability of Human Visual Areas. Cerebral cortex (New York, N.Y. : 1991), 18(2010-2018), 2008.

[48] Y. B. Saalmann, M. A. Pinsk, L. Wang, X. Li, and S. Kastner. The pulvinar regulates information transmission between cortical areas based on attentional demands. Science, 337:753-756, 2012.

[49] R. Salmelin and R. Hari. Characterization of spontaneous MEG rhythms in healthy adults. Electroencephalography and clinical neurophysiology, 91:237-248, 1994.

[50] S. Shipp. The functional logic of cortico-pulvinar connections. Philosophical transactions. Series B, Biological Sciences, 358:1605-1624, 2003.

[51] L.R. Silva, Y. Amitai, and B.W. Connorst. Intrinsic Oscillations of Neocortex Generated by Layer 5 Pyramidal Neurons. Science, 251:432-435, 1991. 
[52] A. C. Snyder and J. J. Foxe. Anticipatory Attentional Suppression of Visual Features Indexed by Oscillatory Alpha-Band Power Increases : A HighDensity Electrical Mapping Study. Journal of Neuroscience, 30(11):40244032, 2010.

[53] E. Spaak, M. Bonnefond, A. Maier, D.A. Leopold, and O. Jensen. Layerspecific entrainment of gamma-band neural activity by the alpha rhythm in monkey visual cortex. Current Biology, 22:2313-2318, 2012.

[54] C.J. Stam, J.P.M. Pijn, P. Suffczynski, and F.H. Lopes da Silva. Dynamics of the human alpha rhythm: evidence for non-linearity? Clinical Neurophysiology, 110(10):1801-1813, October 1999.

[55] N. Tzourio-Mazoyer, B. Landeau, D. Papathanassiou, F. Crivello, O. Etard, N. Delcroix, B. Mazoyer, and M. Joliot. Automated anatomical labeling of activations in SPM using a macroscopic anatomical parcellation of the MNI MRI single-subject Brain. NeuroImage, 289:273-289, 2002.

[56] M. Woolrich, L. Hunt, A. Groves, and G. Barnes. MEG beamforming using Bayesian PCA for adaptive data covariance matrix regularization. NeuroImage, 57(4):1466-1479, 2011.

[57] N. Yamagishi, N. Goda, D. E. Callan, S. J. Anderson, and M. Kawato. Attentional shifts towards an expected visual target alter the level of alphaband oscillatory activity in the human calcarine cortex. Cognitive Brain Research, 25:799-809, 2005. 


\section{Appendices}

\section{A. Model equations}

The state-variables of the $k$-th region $(k=1, \cdots, N)$ are the average membrane potentials of excitatory and inhibitory neurons, denoted by $V_{e}^{k}$ and $V_{i}^{k}$, respectively. They satisfy the following equations:

$$
\begin{aligned}
V_{e}^{k}(t) & =-h_{\mathrm{I}} \otimes\left[\gamma_{i e}^{k} Q_{i}^{k}(t)+\sum_{l \neq k} \beta_{i e}^{l k} Q_{i}^{l}\left(t-\tau_{l, k}\right)\right]+h_{\mathrm{E}} \otimes\left[\sum_{l \neq k} \beta_{e e}^{l k} Q_{e}^{l}\left(t-\tau_{l, k}\right)+p^{k}(t)\right], \\
V_{i}^{k}(t) & =-h_{\mathrm{I}} \otimes\left[\gamma_{i i}^{k} Q_{i}^{k}(t)+\sum_{l \neq k} \beta_{i i}^{l k} Q_{i}^{l}\left(t-\tau_{l, k}\right)\right]+h_{\mathrm{E}} \otimes\left[\sum_{l \neq k} \beta_{e i}^{l k} Q_{e}^{l}\left(t-\tau_{l, k}\right)+\gamma_{e i}^{k} Q_{e}^{k}(t)\right],
\end{aligned}
$$

where $\otimes$ denotes temporal convolution, $h_{\mathrm{E}}$ and $h_{\mathrm{I}}$ denote the average excitatory and inhibitory post-synaptic potentials, respectively, $\gamma_{p q}^{k}$ denotes the number of synaptic contacts on neurons of type $q$ from neurons of type $p$ within region $k$, and $\beta_{p q}^{l k}$ denotes the number of synaptic contacts on neurons of type $q$ within region $k$ from neurons of type $p$ within region $l$. Coupling between region $k$ and $l$ is assumed to have a propagation delay $\tau_{l, k}=\tau_{k, l}$ although all delays are set to zero in the present study, since they have practically no influence on the simulated amplitudes. Furthermore, $Q_{p}^{l}(t)=S\left(V_{p}^{l}(t)\right)$ denotes the average firing-rate of neurons of type $p$ in region $l$ at time $t$. Moreover, $p^{k}=\bar{p}^{k}+$ $\sigma^{k} \xi^{k}(t)$ models the afferent input to region $k$, which is comprised of a stationary firing-rate $\bar{p}^{k}$ and a fluctuating term $\sigma^{k} \xi^{k}(t)$ approximating the complicated afferent input during resting-state conditions by a white-noise process. We assume that the incoming fluctuations to different regions are uncorrelated, that is, $\left\langle\xi^{k} \xi^{l}\right\rangle=\delta_{k l}$. The parameter values are listed in the table 1 . 


\begin{tabular}{lll}
\hline \hline Parameter & Symbol & Nominal value \\
\hline Average spike-threshold & $\rho_{2}$ & $1 \mathrm{mV}$ \\
Dispersion of spike-thresholds & $\rho_{1}$ & $2 \mathrm{mV}^{-1}$ \\
Spike-threshold deviation & $\sigma_{k}$ & $3.3 \mathrm{mV}$ \\
Efficacy of PY $\rightarrow$ IN synapses & $\epsilon_{p p}$ & $4 \mathrm{mV}$ \\
Efficacy of IN $\rightarrow$ PY synapses & $\epsilon_{i p}$ & $32 \mathrm{mV}$ \\
Efficacy of PY $\rightarrow$ TC synapses & $\epsilon_{p t}$ & $8 \mathrm{mV}$ \\
Efficacy of PY $\rightarrow$ RE synapses & $\epsilon_{p r}$ & $0.4 \mathrm{mV}$ \\
Efficacy of TC $\rightarrow$ PY synapses & $\epsilon_{t p}$ & $4 \mathrm{mV}$ \\
Efficacy of RE $\rightarrow$ TC synapses & $\epsilon_{r t}$ & $32 \mathrm{mV}$ \\
Efficacy of TC $\rightarrow$ RE synapses & $\epsilon_{t r}$ & $4 \mathrm{mV}$ \\
Excitatory synaptic rate-constant & $\kappa_{e}$ & $162.5 \mathrm{~s}^{-1}$ \\
Inhibitory synaptic rate-constant & $\kappa_{i}$ & $\kappa_{e} / 4 \mathrm{~s}^{-1}$ \\
Number of synaptic contacts excitatory $\rightarrow$ inhibitory & $\gamma_{1}$ & 64 \\
Number of synaptic contacts inhibitory $\rightarrow$ excitatory & $\gamma_{2}$ & 64 \\
Propagation delay between region $k$ and $l$ & $\tau_{l, k}$ & $0 \mathrm{~s}$ \\
Afferent firing-rate into region $k$ & $\bar{p}^{k}$ & $270 \mathrm{~s}^{-1}$ \\
Standard-deviation of $p$ in region $k$ & $\sigma^{k}$ & $0 \mathrm{or} \mathrm{s}^{-1}$ \\
Global cortico-cortical coupling & $\mathrm{K}_{1}$ & $\geq 0$ \\
Global thalamic feedback & $\mathrm{K}_{2}$ & $\geq 0$
\end{tabular}

Table 2: Descriptions, symbols, and nominal values of the model parameters

\section{B. Linear stability}

Numerical simulations are performed by rewriting the model equations as a system of $N$ 8-dimensional coupled stochastic delay differential equations, which are obtained by rewriting the convolutions $h_{\mathrm{E}} \otimes$ and $h_{\mathrm{I}} \otimes$ as second-order differ- 
ential operators. In this form, the dynamics of region $k$ is governed by

$$
\begin{aligned}
& \dot{V}_{e, 1}^{k}(t)=U_{e, 1}^{k}(t), \\
& \left.\dot{U}_{e, 1}^{k}(t)=-2 \kappa_{\mathrm{E}} U_{e, 1}^{k}-\kappa_{\mathrm{E}}^{2} V_{e, 1}^{k}+\kappa_{\mathrm{E}} H_{\mathrm{E}}\left[p^{k}(t)+\sum_{l \neq k} \beta_{e e}^{l, k} Q_{e}^{l}\left(t-\tau_{l k}\right)\right)\right], \\
& \dot{V}_{e, 2}^{k}(t)=U_{e, 2}^{k}(t), \\
& \left.\dot{U}_{e, 2}^{k}(t)=-2 \kappa_{\mathrm{I}} U_{e, 2}^{k}-\kappa_{\mathrm{I}}^{2} V_{e, 1}^{k}+\kappa_{\mathrm{I}} H_{\mathrm{I}}\left[\gamma_{i e}^{k} Q_{i}^{k}(t)+\sum_{l \neq k} \beta_{e e}^{l, k} Q_{i}^{l}\left(t-\tau_{l k}\right)\right)\right], \\
& \dot{V}_{i, 1}^{k}(t)=U_{i, 1}^{k}(t), \\
& \left.\dot{U}_{i, 1}^{k}(t)=-2 \kappa_{\mathrm{E}} U_{i, 1}^{k}-\kappa_{\mathrm{E}}^{2} V_{i, 1}^{k}+\kappa_{\mathrm{E}} H_{\mathrm{E}}\left[\gamma_{e i}^{k} Q_{e}^{k}(t)+\sum_{l \neq k} \beta_{e i}^{l, k} Q_{e}^{l}\left(t-\tau_{l k}\right)\right)\right], \\
& \dot{V}_{i, 2}^{k}(t)=U_{i, 2}^{k}(t), \\
& \left.\dot{U}_{i, 2}^{k}(t)=-2 \kappa_{\mathrm{I}} U_{i, 2}^{k}-\kappa_{\mathrm{I}}^{2} V_{i, 1}^{k}+\kappa_{\mathrm{I}} H_{\mathrm{I}}\left[\gamma_{i i}^{k} Q_{i}^{k}(t)+\sum_{l \neq k} \beta_{i i}^{l, k} Q_{i}^{l}\left(t-\tau_{l k}\right)\right)\right],
\end{aligned}
$$

where $V_{e}=V_{e, 1}-V_{e, 2}$ and $V_{i}=V_{i, 1}-V_{i, 2}$, and denotes taking the derivative with respect to time $t$. Linear stability was assessed by numerically solving the steady-state equations, which are obtained by setting all derivates to zero, the subsequently computing the system's Jacobian at the steady-state.

\section{Source-strengths}

Assuming the noise-driven fluctuations about the steady-state to be small, the model equations can be linearized about the steady-state and the fluctuations are found to obey the following equations in the Laplace domain:

$$
\begin{aligned}
V_{e}^{k} & =-L_{\mathrm{I}}\left[\gamma_{i e}^{k} G_{i}^{k} V_{i}^{k}+\sum_{l \neq k} \beta_{i e}^{l k} G_{i}^{l} V_{i}^{l}\right]+L_{\mathrm{E}}\left[\sum_{l \neq k} \beta_{e e}^{l k} G_{e}^{l} V_{e}^{l}+\sigma^{k}\right], \\
V_{i}^{k} & =-L_{\mathrm{I}}\left[\gamma_{i i}^{k} G_{i}^{k} V_{i}^{k}+\sum_{l \neq k} \beta_{i i}^{l k} G_{i}^{l} V_{i}^{l} e^{-\tau_{l k} s}\right]+L_{\mathrm{E}}\left[\sum_{l \neq k} \beta_{e i}^{l k} G_{e}^{l} V_{e}^{l} e^{-\tau_{l k} s}+\gamma_{e i}^{k} G_{e}^{k} V_{e}^{k}\right],
\end{aligned}
$$

where

$$
G_{e}^{k}=\frac{d S}{d v}\left(\bar{V}_{e}^{k}\right)
$$

denotes the average gain of excitatory neurons in region $k$ and similarly for inhibitory neurons, and where

$$
L_{\mathrm{E}}(s)=\frac{H_{\mathrm{E}} k_{\mathrm{E}}}{\left(s+k_{\mathrm{E}}\right)^{2}},
$$

denotes the average tranfer function of excitatory synapses and similarly for inhibitory synapses. 
To solve this system of equations for $V=\left(V_{e}^{1}, V_{i}^{1}, \cdots, V_{e}^{N}, V_{i}^{N}\right)^{\prime}$, we write $M_{1}$ for the blockdiagonal matrix with $k$-th block given by

$$
\left(\begin{array}{cc}
0 & -\gamma_{i e}^{k} G_{i}^{k} L_{\mathrm{I}} \\
-\gamma_{e i}^{k} G_{e}^{k} L_{\mathrm{E}} & -\gamma_{i i}^{k} G_{i}^{k} L_{\mathrm{I}}
\end{array}\right)
$$

and $M_{2}$ for the blockmatrix with $(k, l)$-th block given by

$$
\left(\begin{array}{cc}
\beta_{e e}^{l k} G_{e}^{l} L_{\mathrm{E}} & -\beta_{i e}^{l k} G_{i}^{l} L_{\mathrm{I}} \\
\beta_{e i}^{l k} G_{e}^{l} L_{\mathrm{E}} & -\beta_{i i}^{l k} G_{i}^{l} L_{\mathrm{I}}
\end{array}\right) e^{-\tau_{l k} s},
$$

for $l \neq k$. Note that $M_{1}$ and $M_{2}$ relate to the local and global coupling structure, respectively. Furthermore, with $\Sigma=\left(\sigma_{1} L_{\mathrm{E}}, \cdots, \sigma_{1} L_{\mathrm{E}}\right)^{\prime}$ and $M=M_{1}+M_{2}$, the Laplace domain equations can be written in matrixform as

$$
V(s)=M V(s)+\Sigma(s)
$$

from which we obtain

$$
V(s)=(I-M(s))^{-1} \Sigma(s),
$$

where $I$ denotes the identity matrix of size $2 N$. The entries of $V(s)$ correspond to the transfer functions of the excitatory and inhibitory populations in the different regions. If the steady-state is stable, the Fourier spectra of the excitatory populations can be calculated by setting $s=i \omega$. Thus, the Fourier spectra of the MEG signals projected onto the $k$-th region are modeled by

$$
V_{\mathrm{MEG}}^{(k)}(\omega)=V_{e}^{k}(i \omega)
$$

Using the assumption that the afferent fluctuations impinging on the different regions are uncorrelated, the MEG cross-spectral matrix is given by

$$
S_{\mathrm{MEG}}(\omega)=V_{\mathrm{MEG}}(\omega) D_{P}(\omega)\left(V_{\mathrm{MEG}}(\omega) D_{P}(\omega)\right)^{\dagger},
$$

where $D_{P}(\omega)$ denotes the diagonal matrix with the vector $P(\omega)$ as the diagonal and $\dagger$ denotes conjugate-transpose, which can be rewritten as

$$
S_{\mathrm{MEG}}(\omega)=\sigma^{2} V_{\mathrm{MEG}}(\omega) V_{\mathrm{MEG}}(\omega)^{\dagger} .
$$

The strength $A_{k}$ of source-region $k$, as measured by its standard-deviation, is now given by

$$
A_{k}^{2}=\frac{1}{2 \pi} \int_{-\infty}^{\infty} S_{\mathrm{MEG}}^{k}(\omega) d \omega,
$$

where $S_{\mathrm{MEG}}^{k}(\omega)$ denotes the $k$-th entry on the diagonal of $S_{\mathrm{MEG}}(\omega)$. In the calculation of the source-strengths, we sampled $S_{\mathrm{MEG}}^{k}$ between 0 and $40 \mathrm{~Hz}$ with spectral resolution $\Delta \omega=0.1 \mathrm{~Hz}$. 


\section{Functional correlations}

The cross-correlation function $\Gamma_{\text {MEG }}^{k, l}(\tau)$ between the MEG signals at regions $k$ and $l$ at lag $\tau$ is given by the normalized inverse Fourier transform of the cross-spectrum between regions $k$ and $l$

$$
\Gamma_{\mathrm{MEG}}^{k, l}(\tau)=\frac{1}{A_{k} A_{l}} \int_{-\infty}^{\infty} S_{\mathrm{MEG}}^{k, l}(\omega) e^{i \tau \omega} d \omega,
$$

where $S_{\mathrm{MEG}}^{k, l}(\omega)$ denotes the $(k, l)$-th entry of $S_{\mathrm{MEG}}(\omega)$. In particular, the functional connectivity between region $k$ and $l$ as characterized by the correlation coefficient between the corresponding MEG signals is given by $\Gamma_{\text {MEG }}^{k, l}(0)$. In the calculation of the correlation coefficients, we sampled $S_{\mathrm{MEG}}^{k}$ with spectral resolution

$$
\Delta \omega=\frac{2 \pi}{4(2 N+1) 10^{-3}}
$$

$\mathrm{Hz}$, which corresponds to a temporal solution of $\Gamma_{\mathrm{MEG}}^{k, l}$ of

$$
\Delta t=2 \pi / \Delta \omega(2 N+1)
$$

ms. Settting $N=200$, this gives $\Delta t=4 \mathrm{~ms}$ and a maximal observable delay of $\pm 800 \mathrm{~ms}$. 